\title{
SIMULACIÓN VIRTUAL CON TECNOLOGÍA HÁPTICA: ENTRENAMIENTO PARA LA CIRUGÍA DE RODILLA
}

Jesús Alberto Acosta Morón MD*, Edgar Alberto Muñoz Vargas MD**

\section{Resumen}

En cirugía de rodilla, ya sea artroscópica o abierta, se requiere una amplia curva de aprendizaje para garantizar excelentes resultados. Durante años han sido muchos los procesos utilizados desde la observación hasta los medios de realidad virtual. Presentamos un informe preliminar de nuestra experiencia con un sistema de simulación virtual basado en tecnología háptica, para el entrenamiento en los remplazos articulares de rodilla en el Hospital de San José de Bogotá DC. La incursión de la informática en la educación médica y la utilización de simuladores para el aprendizaje de procedimientos quirúrgicos son herramientas claves para las personas que se encuentran en formación. Aunque en la actualidad no hay un estudio con validez estadística para demostrar que este método es el mejor sistema de entrenamiento, en nuestra experiencia la realidad virtual y la tecnología háptica, entre muchos beneficios, facilitan la adquisición de habilidades comunicativas, clínicas y psicomotrices, disminuyen el tiempo necesario para el aprendizaje de habilidades y la experiencia adquirida es fácil transferirla a la realidad.

Palabras clave: háptica, artroscopia, entrenamiento.

\section{VIRTUAL SIMULATION WITH HAPTIC TECHNOLOGY: A TRAINING METHOD FOR KNEE SURGERY}

\begin{abstract}
A broad learning curve is required for knee surgery either conducted by arthroscopy or open surgery to ensure excellent results. During years, many processes have been used, from observation to virtual reality systems. A preliminary report on our experience with a virtual simulation system based on haptic technology to conduct training in knee joint replacement at Hospital de San José de Bogotá DC is herein presented. The incursion of computer systems in medical education and the use of simulators to learn surgical procedures are key tools for the trainees. Although,currently there is no study with statistical validity to demonstrate this method is the best training system, in our experience, virtual reality and haptic technology facilitate the acquisition of communicative, clinical and psychomotor skills, reduce the necessary time to learn skills and expertise acquired is easily transferred to reality.
\end{abstract}

Key words: haptic, arthroscopy, training.

Fecha recibido: julio 18 de 2011 - Fecha aceptado: agosto 19 de 2011

\footnotetext{
Ortopedista, Residente de Cirugia Artroscópica y de Rodilla, Fundación Universitaria de Ciencias de la Salud, Hospital de San José. Bogotá DC. Colombia.
}

Colombia.

** Director del Programa de Artroscopia y Cirugía de Rodilla. Profesor Titular, Vicerrector Académico, Fundación Universitaria de Ciencias de la Salud, Bogotá DC. Colombia. 


\section{Introducción}

En cirugía ortopédica la artroscopia y la artroplastia de rodilla son operaciones estándar que tienen una técnica demandante y necesitan un aprendizaje paso a paso. ${ }^{1}$ El entrenamiento necesario para realizar con eficiencia estos procedimientos requiere además de dedicación, una cuidadosa curva de aprendizaje, la cual desde el principio se basa en la observación y la reproducción del actuar realizado por el docente. La enseñanza debe orientarse no solo a adquirir la habilidad necesaria para la práctica, sino a evitar complicaciones y eventos adversos, como serían el daño iatrogénico del cartílago articular y la lesión de partes blandas en el caso de la cirugía artroscópica, o la mala orientación de los componentes protésicos y la creación de inestabilidades cuando nos referimos a los reemplazos articulares..$^{2-10}$

Por otro lado, es importante anotar que el costo del entrenamiento es alto, como bien lo afirmaron Bridges y Diamond (1999), que solo para las cirugías artroscópicas ascendió a cerca de US\$48.000 en Estados Unidos. ${ }^{3-5}$ El entrenamiento con simuladores quirúrgicos ha ido adquiriendo importancia y popularidad pues estos equipos permiten repetir procesos que no ponen en riesgo la integridad física de los pacientes y sin duda mejoran la habilidad manual y estereoscópica.

El aprendizaje de estas cirugías en cadáveres es una buena opción, con la limitante de ser un método costoso que requiere una amplia infraestructura. Se ha determinado que esta práctica quita responsabilidad y genera pérdida de interés por parte del estudiante, razón por la cual en todos los casos se requiere complementar con modelos artificiales, así como observar videos de cirugías y otros programas de computación. ${ }^{4,5}$

En febrero de 1996, la Academia Americana de Cirugía Ortopédica realizó un curso de instrucción sobre la necesidad de implementar la realidad virtual en el entrenamiento de cirugías de rodilla, dando origen al desarrollo de programas y herramientas con este fin, en diversas sociedades ortopédicas del mundo. ${ }^{5}$ Esto permite inferir que los métodos utilizados para el aprendizaje de las cirugías en la rodilla no habían sido los mejores, por lo que muchos autores han propuesto que este proceso se debe realizar con realidad virtual, pues aseguran que capacita en forma adecuada para la realización de los procedimientos quirúrgicos en cualquier articulación. ${ }^{6-10}$ El desarrollo de esta tecnología va de la mano con la formulación de nuevas técnicas que permitan tener una interacción completa con el medio virtual, creando una retroalimentación que lleve a un ambiente lo más cercano posible a la articulación estudiada.

\section{Desarrollo del equipo}

La primera generación se componía de un miembro inferior con la réplica de una rodilla que permitía movimientos de flexoextensión, más no así de varo y valgo, limitando el aprendizaje de aquellos procedimientos en donde eran necesarios, como la reparación de cuernos meniscales posteriores (tanto mediales como laterales) y además no se podía evaluar el balance ligamentario al simular artroplastias tricompartimentales. Esta deficiencia se incrementaba aún más cuando en estos modelos tampoco se podía realizar rotación interna y externa de la tibia, limitando el entrenamiento de patologías relacionadas con inestabilidades rotacionales. Hoy en día estos inconvenientes han sido resueltos gracias al modelamiento con elementos finitos, los cuales han permitido crear estructuras virtuales con una gran propiedad de realismo e inmersión. ${ }^{5-11}$

En el desarrollo de estas herramientas es donde surge la tecnología háptica, pilar fundamental de nuestro sistema de entrenamiento. El uso de estos elementos virtuales fue registrado en 1997 cuando Gidson ${ }^{6}$ presentó un sistema de simulación para artroscopia de rodilla usando una representación volumétrica y retroalimentación háptica. Este último término se refiere a la información táctil que recibe el aprendiz en un momento real al entrar en interacción con la simulación del procedimiento, con lo cual incrementa las propiedades de simulación con la realidad y, por supuesto, de enseñanza con estos dispositivos. La mayor parte de esta tecnología se desarrolló al principio para el aprendizaje de cirugías artroscópicas y posee propiedades similares con respecto a la utilización de la cámara artroscópica y sus diferentes lentes, además 
de que en gran medida se ha mejorado la interacción con la anatomía real, gracias a la deformación de los tejidos dada mediante una realidad gráfica. ${ }^{5,6-11}$ En Colombia, la Universidad de los Andes ha implementado este tipo de tecnología para simular procedimientos artroscópicos, con un dispositivo háptico que toma como imágenes de información las obtenidas en resonancias magnéticas. ${ }^{12}$

\section{Desarrollo del programa}

En el comienzo, para poder brindar realismo al proceso de entrenamiento se usaron prototipos anatómicos con secciones de $1 \mathrm{~mm}$. Las primeras imágenes fueron tomadas de National Library of Medicine's Visible Human Dataset, las cuales tenían insuficiente resolución. Las bases de datos actuales disponen de diferentes imágenes de origen radiológico, dependiendo del objetivo del entrenamiento. Para los procedimientos artroscópicos se prefiere alimentar el programa con imágenes de resonancia magnética, mientras que para la artroplastia se toman formatos provenientes de bibliotecas médicas, con la posibilidad de realizar reconstrucción tridimensional en modelos óseos con Digital Imaging and Communications in Medicine (DICOM), que pueden ser radiografías, tomografías axiales computarizadas u ortorradiografias, imágenes que son sometidas a modificaciones con mapas de texturas, representación volumétrica y retroalimentación háptica, logrando reproducir la anatomía con una realidad exacta y se trasladan a modelos en $3 \mathrm{D}$, que serán el objetivo sobre el cual se realizará la actividad. ${ }^{13}$

Como resultado del trabajo conjunto entre el Departamento de Ingeniería Mecánica y Mecatrónica de la Universidad Nacional de Colombia y la Fundación Universitaria de Ciencias de la Salud (Hospital de San José), se creó un proyecto para el desarrollo de una herramienta virtual con tecnología háptica, para el aprendizaje de cirugías de reemplazo articular de rodilla. El enfoque inicial ha sido desarrollar un programa con apoyo docente, que le permita simular al residente este procedimiento, mientras se evalúa el progreso de su curva de aprendizaje.
La herramienta permitirá hacer cortes sobre modelos óseos en imagen 3D, similares a los que se realizan en el reemplazo de rodilla; también ofrecerá la oportunidad de elegir el tamaño de los componentes protésicos y su correcta alineación articular. Además posee ayudas educativas que mejorarán la relación docente-alumno, permitiendo que el aprendizaje se haga de una manera integral.

Los objetivos específicos de este proyecto se resumen de la siguiente manera:

- Desarrollar para la docencia de los procedimientos de reemplazos totales de rodilla, la herramienta virtual 3D.

- Caracterizar la curva de aprendizaje con y sin el uso de la herramienta propuesta.

- Explorar entre la población de residentes el impacto de este tipo de entrenador médico.

Para lograr estos objetivos, se creó esta metodología:

\section{Herramienta docente:}

- Visualización de imágenes radiográficas en formatos DICOM, en planos anatómicos diferentes.

- Posibilidad de cortes sobres estas imágenes, similares a los que se realizan en la artroplastia de rodilla (Figura 1).

- Reconstrucción de tejidos específicos con visualización tridimensional.

- Moldeamiento virtual de herramientas y prótesis.

- Análisis del punto de contacto entre prótesis y hueso.

- Herramienta de administración de aprendizaje y evaluación según el nivel del alumno.

\section{Curva de aprendizaje:}

- Realizar sesiones de entrenamiento con los residentes. 

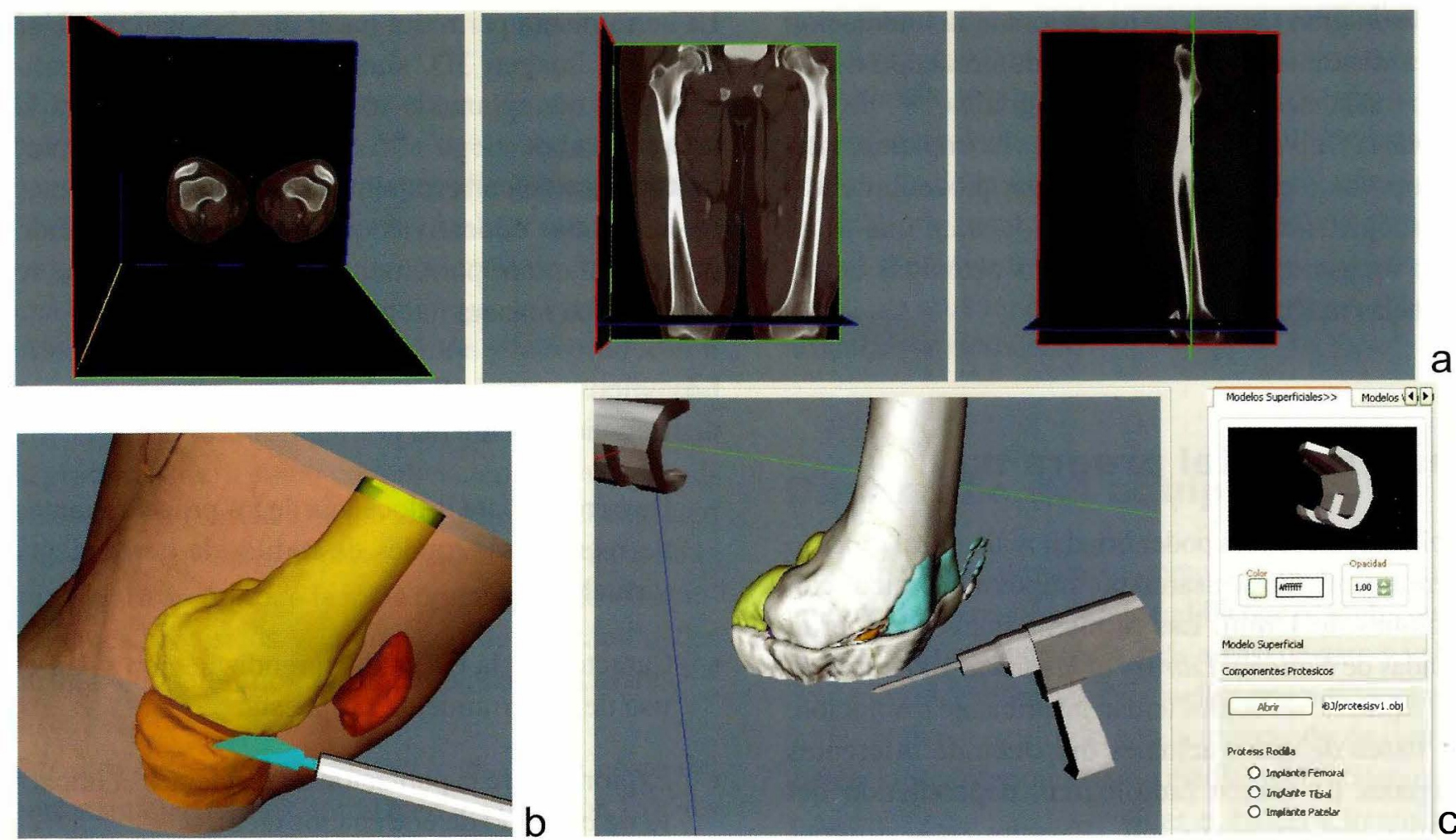

Figura I. Herramientas del entrenador: a) visualización y procesamiento de imágenes médicas,

b) reconstrucción de modelos 3D y c) integración con modelos de prótesis.

- Desarrollar evaluaciones periódicas que permitan conocer el avance del aprendizaje.

- Cuantificar la curva de aprendizaje.

\section{Herramienta háptica:}

- Realimentación de fuerzas en la exploración mediante la interfaz háptica Phanton Omni $®$ de SenSable Inc. (Figura 2).

- Desarrollo de simulación de cortes e interacción con eliminación de partículas y representación de fuerzas en función de las superficies que entran en contacto.

El desarrollo de la herramienta docente y del modelo háptico utilizado en este proyecto se debe a un proyecto de investigación de la Universidad Nacional de Colombia titulado Modelado de tejidos blandos en un simulador háptico para entrenamiento de operaciones en remplazos totales de cadera y de rodilla. Logradas estas herramientas, se desarrollaron las fases de integración, pruebas preliminares, navegación virtual y ajuste de evaluación a residentes y cirujanos de rodilla.

La realidad virtual y la construcción de simuladores para las cirugías articulares es una nueva fuente de aprendizaje para quienes se encuentran en entrenamiento. En la actualidad no hay estudios con significativa validez estadística que demuestren que la aplicación de esta metodología brinda mejores resultados que los actuales métodos de aprendizaje, en especial en países en vía de desarrollo.

Nuestra experiencia con este tipo de tecnología se inició en 2010 mediante la familiarización con estos equipos. Una vez comprendido el funcionamiento de la herramienta, se hicieron las primeras actividades correspondientes a cirugías artroscópicas en modelos de rodillas encaminadas a orientación, ubicación en el plano espacial y triangulación intraarticular. Además, se desarrolló el programa para nuestro proyecto final, 

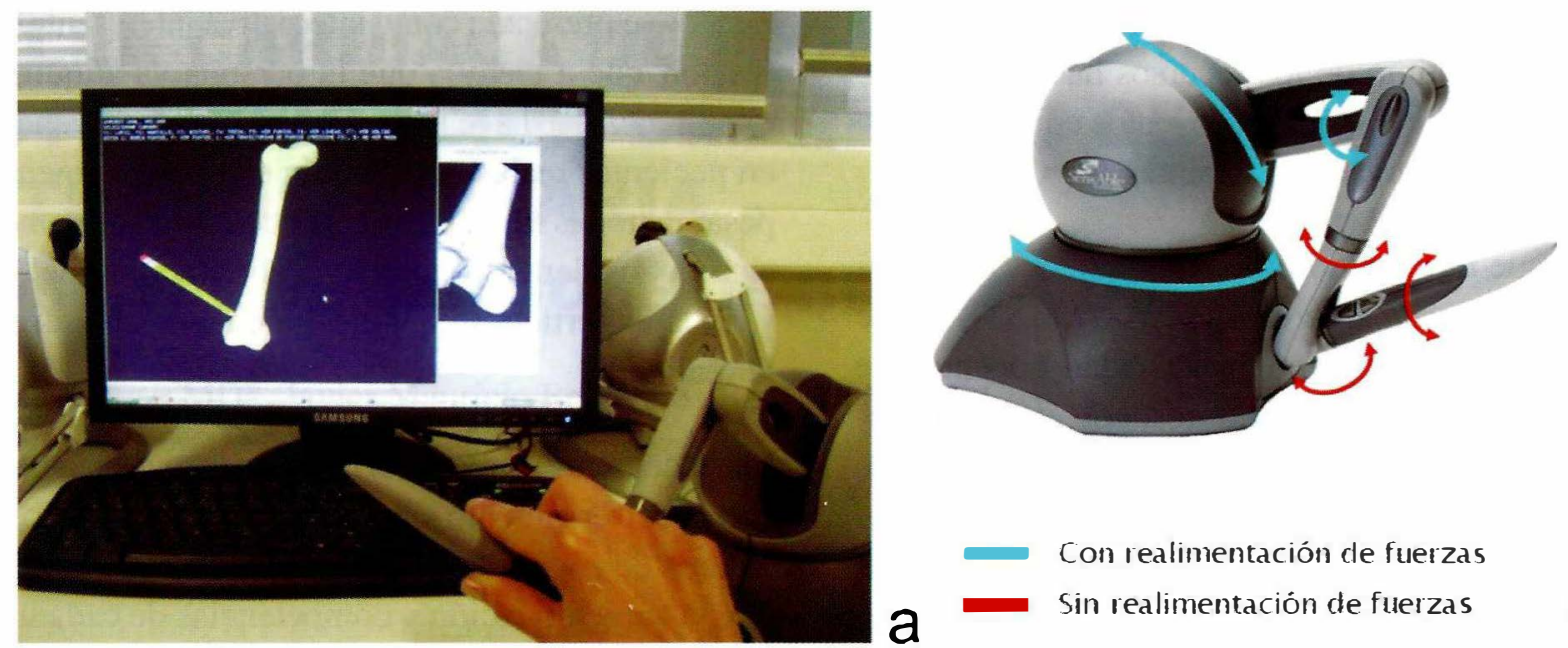

Con realimentación de fuerzas

a

- Sin realimentación de fuerzas

Figura 2. Sistema háptico.a) exploración virtual. b) interfaz Phantom Omni®.

que está centrado en la simulación con realidad háptica para realizar remplazos articulares de rodilla, por lo que podremos expresar nuestra experiencia preliminar con esta herramienta.

Uno de los primeros beneficios con estos simuladores es el reconocimiento de la anatomía, lo cual es indispensable para realizar en forma adecuada cualquier tipo de cirugía. Los procedimientos quirúrgicos, para tener un resultado más preciso y predecible, requieren constancia, repetición y automatización. Pensamos que estos requisitos se pueden aplicar en la práctica quirúrgica cuando haya familiarización con los procedimientos practicados en el simulador.

El contacto físico inicial con los equipos de simulación se realiza de manera similar a la exposición que tenemos con los videojuegos, constituidos por una interfase en un monitor, en el que se elige el tipo de actividad a realizar y el sistema nos informa nuestra progresión en cada uno de los procesos evaluados por el programa. Un reporte del Instituto de Tecnología Médica Avanzada y del Centro Médico Israelí de Nueva York, concluyó que los cirujanos que habían estado en contacto con videojuegos presentaron mejores resultados (40\%) al momento de hacer suturas laparoscópicas en simuladores, que quienes nunca lo habían hecho. ${ }^{14}$

La progresión en el aprendizaje es posible gracias a los objetivos, que a medida que el estudiante los va alcan- zando, se van incrementando en complejidad en forma automática, para seguir con ejercicios cada vez más laboriosos y que requieren mayor habilidad y destreza.

En el momento de pasar de procedimientos simulados a cirugías reales, se percibe que hay una mayor familiaridad con el instrumental utilizado y se activa el mapa mental conceptual de la técnica quirúrgica mejorando el sentido estereoscópico. Además se adquiere confianza, estar atento a los pasos a seguir en una cirugía que requiere mayor delicadeza y concentración y, a medida que se incrementan las horas de entrenamiento, se nota una disminución en el tiempo operatorio y se corrigen o evitan pasos innecesarios. Por estas razones pensamos que la simulación en la cirugía de la rodilla tiene una gran aplicación docente.

Sabemos que las ayudantías quirúrgicas y las cirugías supervisadas por expertos son posibles hoy en todos los centros de entrenamiento de nuestro país, mientras que el uso de simuladores no se ha generalizado en nuestras Facultades de Medicina, debido a que son menos accesibles, requieren infraestructura compleja y aún no ofrecen una buena relación costo/beneficio.

Con la inclusión de la informática en la renovación de la educación médica, el entrenamiento con simuladores junto con el aprendizaje por medio de portales interactivos en internet, constituyen piezas clave para lograr rapidez y eficacia en las curvas de aprendizaje. Son mé- 
todos éticos y seguros para los pacientes. La necesidad de un manejo óptimo de los recursos hace imprescindible su incorporación en los proyectos educacionales, como elemento innovador y garantía de éxito.

Tanto es, que ha surgido un nuevo concepto: Educación Médica Basada en la Simulación (EMBS), que se puede definir como cualquier actividad docente que utilice la ayuda de los simuladores, con el fin de estimular y favorecer el aprendizaje, creando en lo posible un escenario clínico. La EMBS facilita al profesional la adquisición de habilidades comunicativas, clínicas y psicomotrices.

Otras ventajas educativas en el entrenamiento basado en simulación son las siguientes:

- Disminuye el tiempo necesario para el aprendizaje de habilidades.

- Es una formación guiada por el error, que permite llegar hasta las últimas consecuencias, sin ninguna repercusión real.

- Es personalizado, es decir, de acuerdo con las habilidades del que aprende, según su ritmo y rendimiento.

- La experiencia adquirida con este método es fácil de transferir a la realidad.

Es muy probable que en el futuro cada programa académico de ortopedia y traumatología exija al residente un contacto inicial con sistemas de entrenamiento, antes del primer abordaje quirúrgico en un paciente real en la sala de cirugía. Las unidades de entrenamiento con simulación se incorporarán a los hospitales y demás centros de salud. Se dejará de ver en las instituciones universitarias al paciente como un elemento de enseñanza, permitirá el ahorro de recursos (al acortar la duración del procedimiento), disminuirá el mal uso del instrumental y, por otro lado, habrá una posible reducción de demandas por mala praxis e inexperiencia.

Los simuladores y la tecnología háptica, según nuestros resultados, son excelentes herramientas que permiten el entrenamiento de determinadas maniobras propias del transcurrir de una cirugía y en estos mo- mentos se presentan como alternativa interesante. Cabe resaltar que a medida que la persona va adquiriendo experiencia, se hace necesario realizar intervenciones en pacientes reales, ya que aún en estos momentos, a pesar de todas las herramientas de que disponemos, se sigue considerando que la prueba máxima del saber y de la experticia se demuestra cuando se enfrenta a la realidad.

Más adelante estaremos brindando la experiencia final con el entrenamiento simulado de los reemplazos articulares de rodilla en el Hospital de San José, al igual que la caracterización y competencias de cada uno de los miembros de los diferentes niveles del servicio que intervienen en la cirugía artroscópica y de rodilla.

\section{Referencias}

1. Miller WE. Learning arthroscopy. South Med J. 1985; 78(8): 935-37.

2. Sherman KP, Ward JW, Wills DP, Mohsen AM. A portable virtual environment knee arthroscopy training system with objective scoring. Stud Health Technol Inform. 1999; 62: 335-36.

3. Bridges M, Diamond DL. The financial impact of teaching surgical residents in the operating room. Am. J Surg. 1999; 177(1): 28-32.

4. Meyer RD, Tamarapalli JR, Lemons JE. Arthroscopy training using a "black box" technique. Arthroscopy. 1993; 9(3): 338-40.

5. Cannon WD, Eckhoff DG, Garrett WE Jr., Hunter RE, Sweeney HJ. Report of a group developing a virtual reality simulator for arthroscopic surgery of the knee joint. Clin Orthop Relat Res. 2006 Jan; 442:21-9.

6. Gibson S, Samosky J, Mor A, Fyock C, Grimson E, Kanade T, et al. Simulating arthroscopic knee surgery using volumetric object representations, real-time volume rendering and haptic feedback. Lect Notes Comput S. 1997; 1205(1): 369-78.

7. Heng P-A, Cheng CY, Wong TT, Xu Y, Chui YP, Chan KM, Tso SK. A VirtualReality training system for knee arthroscopy surgery. IEEE Trans Inf Technol Biomed. 2004; 8(2): 217-227.

8. Mabrey J, Gillogly S, Kasser J, Sweeney HJ, Zarins B, Melvis H, et al. Virtual reality simulation of arthroscopy of the knee. Arthroscopy. 2002 JulAug;18(6):E28

9. Ward JW, Willis DPM, Sherman KP, Mohsen AMMA. The development of an arthroscopic surgical simulator with haptic feedback. Future Gener Comp Syst. 1998; 14(3-4): 243-51

10. McCarthy AD, Moody L, Waterworth AR, Bickerstaff DR. Passive haptics in a knee arthroscopy simulator Is It Valid for Core Skills Training? Clin Orthop Relat Res. 2006 Jan; 442:13-20.

11. Pinto ML, Sabater JM, Sofrony J, Badesa J, Rodriguez J, Garcia N. Haptic simulator for training of total knee replacement. In: Biomedical robotics and biomechatronics (BioRob). 3rd ed. Tokyo: IEEE; 2010. p 221-26.

12. Herrera O, Espitia R, Zared G, Figueroa P, Torres J, García A. Desarrollo de un sistema de entrenamiento médico para artroscopia de rodilla. Rev. Col. Or. Tra. 2008 sep; 22(3): 172-77.

13. Ding H, Liang T, Wang G, Liu W. Virtual Total Knee Replacement system based on VTK. In: Proceedings of the 23th International Conference on Biomedical Engineering; 2008; Singapore : Springer; 2009. p. 1028-1031.

14. Morris BR. Pre-op warm-up: a few videogames. New York Times. 2004 Feb 26; sect G: 4 . 